\title{
Verwaltungsdokumente als Vehikel in der biografischen Auseinandersetzung mit der Heimerziehung der 1950er und 1960er Jahre
}

\author{
Magdalena Apel
}

Online publiziert: 20. Januar 2020

(C) Der/die Autor(en) 2019

Zusammenfassung In dem Beitrag wird die Bedeutung von Fürsorgeakten der 1950er und 1960er Jahre für Zeitzeug_innen der damaligen Heimerziehung herausgearbeitet. Dafür werden biografisch-narrative Interviews mit einer hermeneutischen Perspektive analysiert. Die Bedeutung der Dokumente im Rahmen biografischer Aufarbeitung von vergangenen Geschehnissen steht dabei im Zentrum. Der Beitrag zeigt, dass die Wirkmacht der Schriftstücke für die befragten Zeitzeug_innen von der Vergangenheit bis in die Gegenwart reicht. Akteur_innen der Heimerziehung sowie von diesen in den Akten festgehaltene Zuschreibungen an die ehemals im Heim Untergebrachten werden in den Erzählungen aus der Retrospektive der Interviewten wieder gegenwärtig. Der Akteninhalt kann Antworten auf lange gestellte, identitätsstiftende Fragen liefern, aber auch mit der eigenen Erinnerung in heftigen Widerspruch treten. Der weitere Umgang mit den Dokumenten im Rahmen der eigenen biografischen Auseinandersetzung wird von den Zeitzeug_innen dementsprechend unterschiedlich imaginiert und ausgestaltet.

\section{Administrative Documents as a Vehicle in the Biographical Processing of Residential Care in the 1950s and 1960s}

\footnotetext{
M. Apel $(\bowtie)$

FB 01 Humanwissenschaften, Institut für Sozialwesen, Universität Kassel,

Arnold-Bode-Straße 10, 34109 Kassel, Deutschland

E-Mail: apel@uni-kassel.de
}

Abstract The paper highlights the importance of administrative documents, generated in the 1950s and 1960s, for contemporary witnesses of former residential care. To this purpose, biographical-narrative interviews are examined from a hermeneutical perspective with the significance of the documents in the context of autobiographical analysis of the past constituting the focus of this article. The paper shows that for the interviewees the documents have the power to bring the past to the 
present. Persons formerly responsible for residential care and the attributions noted in the files become present again in the retrospective narratives of the interviewees. The content of the files can provide answers to identity-forming questions which have been asked for a long time, but it can also violently contradict their own memory. Therefore, the contemporary witnesses differently imagine and shape the further handling of the documents in the context of their own biographical processing.

\section{Einleitung}

Zeitzeug_innen, die in den Jahren 1949 bis 1975 in westdeutschen Fürsorgeheimen untergebracht waren, werden spätestens seit der Anhörung Betroffener vor dem Petitionsausschuss des Deutschen Bundestages ab 2006 vermehrt mit einem medialen Diskurs konfrontiert, der sich um die Aufarbeitung der damaligen Fürsorgepraxis dreht. Die Heimerziehung der 1950er und 1960er Jahre zielte auf die oftmals mit psychischer und physischer Gewalt durchgeführte Disziplinierung von Kindern und Jugendlichen, die den damals vorherrschenden Normalitäts- und Ordnungsvorstellungen nicht entsprachen (vgl. z.B. Runder Tisch Heimerziehung 2010; Bereswill et al. 2013). Aufgrund der Initiative Betroffener wurde die Situation in den damaligen Heimen vom Petitionsausschuss des Deutschen Bundestages untersucht, kritisiert und als erfahrenes Unrecht anerkannt. Infolgedessen hatte der 2009 etablierte „Runde Tisch Heimerziehung in den 50er und 60er Jahren“, der sich aus Vertreter_innen verschiedener Institutionen und Personengruppen ${ }^{1}$ zusammensetzte, zur Aufgabe, die damaligen Geschehnisse aufzuarbeiten und Leidtragende bei der individuellen Beschäftigung mit ihren Heimerfahrungen und der Wahrnehmung ihrer Rechte zu unterstützen. In seinem Abschlussbericht schreibt der Runde Tisch Heimerziehung (2010, S. 33), dass ,hierfür insbesondere die Einsicht der Betroffenen in ihre damaligen personenbezogenen Akten sehr hilfreich sein kann“" und gibt eine „Empfehlung [...] zur Akteneinsicht durch ehemalige Heimkinder“ (ebd., S. XVII) heraus. Die eigene Akte könne, laut dieser Empfehlung, Antworten auf identitätsstiftende Fragen liefern und Informationen zu Kindheit und Jugend, Familie und Herkunft sowie zu Umständen der Heimeinweisung oder Dauer der Unterbringung bereithalten. Zudem seien dort möglicherweise rechtlich relevante Belege, die z. B. ein Recht auf Rente und/oder Entschädigungszahlungen dokumentieren könnten, zu finden. Darüber hinaus sei durch die Akteneinsicht ein Einblick in die Meinung der Erziehungsverantwortlichen sowie in die „Probleme“, die den Untergebrachten unterstellt oder vorgeworfen wurden (ebd., S. XVIII) möglich. Damit bestärkte der Runde Tisch Heimerziehung Betroffene in einer aktiven Auseinandersetzung mit ihrer Vergangenheit und den in dieser Zeit entstandenen Dokumenten. Der Weg dahin wurde geebnet, indem man an alle zuständigen Institutionen ,einen dringenden Appell“" richtete, die Akteneinsicht zu ermöglichen (ebd.).

\footnotetext{
1 Für eine ausführliche Auflistung s. Runder Tisch Heimerziehung (2010, S. 5).
} 
Vor diesem Hintergrund verwundert es nicht, dass auch die Zeitzeug_innen, die sich im Rahmen von zwei hier herangezogenen qualitativen Studien ${ }^{2}$ bereit erklärt haben, biografische Interviews zu geben, den gesellschaftlichen Diskurs meist gut kennen und ihn teilweise selbst aktiv mitgestaltet haben. Außerdem haben mehrere Interviewte im Zuge der Auseinandersetzung mit eigenen Institutionserfahrungen ihr Recht auf Akteneinsicht wahrgenommen und die über sie angelegten Verwaltungsdokumente bereits eingesehen.

Die in den 1950er und 1960er Jahren über die in Heimen untergebrachten Kinder und Jugendlichen angelegten so genannten Fürsorgeakten sind heute nur noch partiell in Archiven erhalten und wurden von den jeweiligen Heimen sehr heterogen geführt. Sie dienten der Kommunikation zwischen beteiligten Instanzen der Sozialbürokratie und der Rechtfertigung von verwaltungsrechtlichen Vorgängen. In der Regel finden sich in diesen Akten Schriftstücke wie Einweisungsdokumente, Gerichtsbeschlüsse, pädagogische, psychologische oder psychiatrische Gutachten, Briefwechsel zwischen den zuständigen Behörden oder zwischen Untergebrachten und deren Angehörigen sowie Berichte, um Maßnahmen des Heims zu dokumentieren und zu legitimieren. Die Akten sind Teil der sozialen Kontrolle und Überwachung, die in der Praxis der Heime eine zentrale Bedeutung hatten, und können als Ort von Deutungen, Erklärungen, Prognosen und Begründungen verstanden werden (Apel und Eckhardt 2019).

Für die interviewten Zeitzeug_innen spielt die retrospektive Beschäftigung mit den über sie angelegten Fürsorgeakten im Zusammenhang mit dem Rekonstruieren und Verstehen des eigenen biografischen Gewordenseins eine wichtige Rolle. Die Auseinandersetzung mit der Akte wird so zum Vehikel in der biografischen Aufarbeitung. Die hohe Bedeutung, die der Akteninhalt damit gewinnt, ist in zweierlei Hinsicht problematisch: Erstens gerät dadurch aus dem Blick, dass im bürokratischen Herstellungsprozess der Akten biografische Verläufe nicht nur durch verschiedene Instanzen dokumentiert, sondern dadurch überhaupt erst interaktiv hergestellt, strukturiert und geformt werden (Bereswill und Müller 2018). Zweitens sind Verwaltungsdokumente lückenhaft und können nur einen Bruchteil biografischer Informationen liefern. Auch wenn Biografien grundsätzlich nie vollständig und objektiven Tatsachen entsprechend festgehalten und wiedergegeben werden können, da es sich um subjektive, von verschiedenen Institutionen und Temporalitäten geprägte Perspektiven handelt, suggeriert die Möglichkeit der Akteneinsicht den Zeitzeug_innen, durch die Akte einen Einblick in ihr ,,tatsächlich“ gelebtes Leben erhalten zu können. Diese Fiktion einer rekonstruierbaren Biografie durch Verwaltungsdokumente kann besonders dann in den Vordergrund rücken, wenn andere Anknüpfungspunkte an die Vergangenheit wie z. B. Familienerzählungen fehlen.

Obwohl „die eigene Akte“, verstanden als ein Konglomerat aus allen Schriftstücken, die über eine in Fürsorgeheimen untergebrachte Person angefertigt wurden, den Betroffenen nicht gehört, wird in diesem Beitrag dennoch aus einem bestimmten

\footnotetext{
2 Apel, M. (laufendes Promotionsprojekt): Verkörperung von Ordnung. Eine qualitative Studie der Heimerziehung der 1950er und 1960er Jahre und Bereswill, M.; Höynck, T. (09/2011-08/2012): Heimerziehung in Jugend- und Erziehungsheimen des Landeswohlfahrtsverbands Hessen in den Jahren 1953-1973. Interdisziplinäres Forschungs- und Ausstellungsprojekt.
} 
Grund das Possessivpronomen verwendet: Die Betroffenen haben teilweise immense bürokratische Hürden überwinden müssen, um eine Kopie der über sie angelegten Dokumente zu erhalten und die Akte somit zumindest zum Teil zu ihrer eigenen zu machen. Es wird also der Aneignungspraxis der Zeitzeug_innen gefolgt, die darum kämpfen, dass die in den Akten enthaltenen Informationen ihnen gehören. Der Begriff des Vehikels wurde gewählt, da er nicht nur erlaubt, die Akten als Hilfsmittel oder Instrument bei der biografischen Aufarbeitung zu verstehen, sondern das Bild eines alten, möglicherweise nicht voll funktionsfähigen Fortbewegungsmittels auch die Materialität von Akten sowie den ideellen Wunsch der Interviewten nach persönlichem Fortkommen einfängt. Das sprachliche Bild hat allerdings seine Grenzen, weil angenommen werden könnte, dass mit einer modernisierten Art von Dokumentation biografische Aufarbeitung besser gelingen könnte, was nach den vorangestellten Überlegungen jedoch zu bezweifeln ist.

Vor diesem Hintergrund steht die Frage, welchen Platz Verwaltungsdokumente und die darin enthaltenen Narrative in der Auseinandersetzung mit biografischen Erfahrungen einnehmen können und welche Auswirkungen die individuelle Beschäftigung mit diesen Schriftstücken auf die retrospektive Narration ehemals in Fürsorgeheimen Untergebrachter haben, im Fokus des vorliegenden Beitrages.

Einleitend werden die Datengrundlage sowie das methodische Vorgehen skizziert (2). Im Anschluss werden anhand ausgewählter Interviewsequenzen Erzählungen über den Weg zur eigenen Akte (3), über die Akteneinsicht (4) sowie Handlungs(un)möglichkeiten im Umgang mit der Akte (5) rekonstruiert und analysiert. Im Fazit und Ausblick des Beitrages (6) werden zentrale Ergebnisse der Analyse zusammengefasst und theoretisch eingeordnet.

\section{Datengrundlage und methodisches Vorgehen}

Die hier zitierten Interviewsequenzen stammen aus einem Datenkorpus von insgesamt achtzehn biografisch-narrativen Interviews mit ehemals in Fürsorgeheimen Untergebrachten, die im Rahmen zweier Forschungsprojekte (s. Fußnote 2) erhoben wurden. Die impulsgebende Eingangsfrage war bei beiden Studien so konzipiert, dass die Erfahrungen mit der damaligen Heimerziehung im Zentrum der Erzählungen stehen sollten. Den Interviewten wurde aber auch Raum gegeben, über die Zeit vor und nach dem Heimaufenthalt sowie über ihre aktuelle Lebenssituation zu erzählen. Daraus, und vor dem Hintergrund des oben skizzierten öffentlichen Diskurses um die Aufarbeitung der Heimerziehung der 1950er und 1960er Jahre, ergab es sich im Interviewsetting, dass Zeitzeug_innen die eigene Akte von sich aus zum Thema machten. Teilweise hatten sie zu diesem Zeitpunkt die Dokumente bereits eingesehen, planten dies oder waren an bürokratischen Hürden gescheitert. Die Rekonstruktion der eigenen Biografie gehört für viele Betroffene der Fürsorgeerziehung zum Verarbeitungsprozess des Erlebten. Die eigene Akte bzw. alle noch auffindbaren Dokumente aus der Zeit der Heimerziehung dienen dabei als Vehikel und spielen deshalb eine wichtige Rolle in den Interviews. Wie der Akteninhalt von den Interviewten aus der Retrospektive gelesen wird und welche Bedeutung der Akte in der individuellen Auseinandersetzung mit Institutionserfahrungen zugeschrieben wird, 
waren zentrale Fragestellungen bei der Auswertung der Interviews. Diese erfolgte in Anlehnung an die Methodik der Objektiven Hermeneutik (Oevermann et al. 1979), die mithilfe von Sequenzanalysen die Rekonstruktion des latenten Sinns im Datenmaterial ermöglicht. In den nachfolgenden Abschnitten werden Interviewsequenzen thematisch strukturiert vorgestellt, analysiert und mögliche Lesarten angeboten.

\section{Der Weg zur eigenen Akte}

Dokumente zu lesen, die von kirchlichen oder staatlichen Bediensteten vor mehreren Jahrzenten über das eigene Sein als Kind oder Jugendliche_r verfasst wurden und die das Potenzial hatten, den Lebensweg grundlegend mitzubestimmen, stellt Betroffene vor mehrere Herausforderungen. Zunächst müssen sie sich die Frage stellen, ob sie diese Dokumente überhaupt sehen möchten. Die Zeitzeugin Gisela Heller ${ }^{3}$ erzählt, dass es ,lange gedauert [hat] ganz ganz lange“ bis sie sich dazu entscheiden konnte, ihre Akte einsehen zu wollen. Wie es dazu kam, erläutert sie in der folgenden Sequenz:

vor zwei oder vor drei Jahren hat das äh äh angefangen bei mir dass ich gedacht hab ,äh Gisela“ - da gabs dann ich glaub in der Tageszeitung den ersten Bericht das war auch n Heimkind die hat da s erste Mal ganz öffentlich - die hab ich dafür bewundert obwohl ich hätts nich gemacht ich hätte mein Gesicht nich in die Zeitung gesetzt ähm aber vielleicht bin ich doch auch ne feige Socke ich weiß es ja nich da hab ich gedacht also d du musst dich auch mal da drum kümmern irgendwer muss das ja mal aufarbeiten und dann gab es in dieser Zeitung so ne Adresse

Frau Heller beschreibt hier wie sie durch die zu dieser Zeit noch recht neue mediale Präsenz ehemals im Heim Untergebrachter die Idee entwickelt, sich mit ihrer Heimgeschichte zu beschäftigen. Zum einen bewundert sie die Frau, die ihre Geschichte öffentlich macht und beschreibt dies auch als ausschlaggebend für ihre eigene Entscheidung. Zum anderen reflektiert sie, dass sie ihre Anonymität nicht dafür aufgegeben hätte und bezeichnet sich deswegen als „feige“. In dieser Ambivalenz lässt sich die Angst vor erneuter Stigmatisierung als Heimkind erkennen, aber auch der gesellschaftliche Druck zur Verantwortungsübernahme, den die öffentliche Berichterstattung mit sich bringt. Es ist denkbar, dass aus Letzterem, dem allgemeinen Interesse an der Rekonstruktion eigener biografischer Verläufe, sowie der Identifizierung mit der Frau, die über ihre Heimerfahrung spricht, der Mut resultiert, den sie aufwendet, um die in der Zeitung angegebene Adresse zu kontaktieren.

In einem Telefonat erfährt sie dann von einem Mitarbeiter einer Anlaufstelle für Betroffene, dass derzeit weiter nach Unterlagen gesucht würde. Daraufhin erzählt sie diesem, dass und warum ihr wichtige Zeugnisse von damals fehlen. Der Mitarbeiter bietet ihr Hilfe an, die er aber letztlich nicht leistet:

\footnotetext{
3 Die Namen der Interviewten wurden anonymisiert.
} 
das hatt ich dem da alles erklärt und der hat sich bei mir nie wieder gemeldet und dann war bei mir auch mal ne Zeit lang wo ich gedacht hab ,wer weiß Gisela erzähl nit so viel du weißt do[ch]“- hab ich au gedacht das war vielleicht nit richtig was du da gemacht hast und dann wollte der mir auch Unterlagen schicken weil es ja um diese Entschädigung ging aber hab ich nie von ihm gekriegt und dann hab ich gedacht ,na ja Gisela dann musst du jetz entweder n bisschen vorsichtiger sein oder (seufzt) lässt es auf sich beruhen“"

In dieser Passage wird deutlich, dass Frau Heller gegenüber der Person, die ihr falsche Versprechungen gemacht hat, oder allgemein gegenüber Behörden misstrauisch wird. Sie zweifelt an sich selbst und befürchtet, zu viel erzählt zu haben. Eine Begründung dafür deutet Frau Heller in dem Satzteil „du weißt do[ch]“ an, führt ihren Gedanken aber nicht zu Ende. Es wird zwar nicht klar, was Frau Heller weiß, aber ihre Aussage deutet darauf hin, dass sie bereits schlechte Erfahrungen damit gemacht hat, anderen von ihrer Heimgeschichte zu erzählen. Dies bestätigt sich im weiteren Verlauf des Interviews, wenn Frau Heller erzählt, dass ihr Mann negativ auf die Erzählung ihrer Geschichte reagiert hat und sie danach abwertend behandelt. Die Stigmatisierung ,ehemaliges Heimkind“ schreibt sich damit weiter fort und Frau Hellers Befürchtungen könnten darin begründet sein, vor anderen bloßgestellt zu werden. Möglicherweise ist der Abbruch des Satzes dadurch zu erklären, dass sie auch der Interviewerin vor diesem Hintergrund nicht ausreichend vertraut. Auch wenn Frau Heller den Akteninhalt zu diesem Zeitpunkt noch nicht kennt, erscheint er ihr privat und schützenswert. Dies könnte damit zusammenhängen, dass vielen Kindern und Jugendlichen bereits während der Zeit ihrer Unterbringung bewusst war, dass Dokumente über sie angelegt wurden. Dieses Wissen um „die Akte“ diente in der Heimerziehung nicht zuletzt als Disziplinar- und Kontrollmaßnahme und die damit verbundene Wirkmacht scheint über die Jahre erhalten geblieben. ${ }^{4}$

Bemerkenswert an dieser Sequenz ist außerdem, dass Frau Heller nicht verärgert zu sein scheint. Sie zieht aus der Erfahrung mit dem Mitarbeiter lediglich die Konsequenz, dass sie entweder vorsichtiger sein oder die Suche nach den über sie angefertigten Dokumenten aufgeben muss. Hier könnte die Angst vor Zurückweisung vor den nahe liegenden Ärger über einen unzuverlässigen Vertreter der Verwaltung treten. Nachdem sich immer mehr Betroffene zu Wort melden und der Runde Tisch Heimerziehung entsteht, wächst jedoch Frau Hellers Motivation, sich mit ihrer Vergangenheit zu beschäftigen:

aber dann gabs ja diesen Runden Tisch und das hab ich dann eben verfolgt und dann hab ich gedacht „Gisela du musst dich mal drum kümmern“ aber hm es is nich so einfach den Telefonhörer in die Hand zu nehmen und da dann anzurufen da - da müssen Sie selber den - den Tag erwischen und äh wenn Sie abends um sieben mutig sind nützt Ihnen das nix weil da sitzt niemand mehr im Büro ja und so - doch das hat schon -ne Zeit gedauert ich denke ma so zwei drei Jahre bin ich damit schwanger gegangen mich da ernsthaft mit zu beschäftigen

\footnotetext{
${ }^{4}$ Zur Wirkmacht der Akte während der Heimunterbringung vgl. Apel und Eckhardt (2019). Zur Formung von biografischen Prozessen durch Verwaltungsbürokratie s. Bereswill und Müller (2018). Grundlegend zur Herstellung eines so genannten Zöglings durch Dokumentation siehe Zaft (2011).
} 
Die Einrichtung des Runden Tisches Heimerziehung vergrößerte das öffentliche Interesse und damit auch die Möglichkeit, dass Zeitzeug_innen wie Gisela Heller aktiv werden. Frau Heller spricht über die Beschäftigung mit ihrer Vergangenheit wie über eine Aufgabe, die es abzuarbeiten gilt. Das von ihr verwendete Verb „müssen“ deutet auf einen Zwang hin, der aufgrund der angenommenen gesellschaftlichen Erwartungshaltung und einem daraus resultierenden Gefühl der inneren Verpflichtung Frau Heller dazu bewegt, sich mit ihrer Heimerfahrung auseinanderzusetzen. Bis Frau Heller jedoch tatsächlich aktiv wird, dauert es mehrere Jahre, in denen sie mit der Idee „schwanger gegangen“ ist. Dies könnte bedeuten, dass der Gedanke erst wachsen und ein Teil von ihr werden musste, um sie handlungsfähig zu machen. Wenn vorher Mut aufgebracht werden muss, so wie die Zeitzeugin es beschreibt, kann davon ausgegangen werden, dass mögliche Konsequenzen des eigenen Handelns als potenziell bedrohlich eingestuft werden. Frau Heller hat zum Zeitpunkt des Interviews die über sie angelegte Akte zwar noch nicht gesehen, aber bereits mehrere Schritte unternommen, um sich Zugang zu verschaffen. In dieser Sequenz wird deutlich, dass biografische Aufarbeitung ein Prozess ist, der Zeit zum Nachdenken und Verarbeiten braucht und nicht linear verläuft. Der benötigte Mut, weitere Schritte zu unternehmen, ist auch nach langjähriger Beschäftigung mit dem Thema nicht kontinuierlich vorhanden, sondern lediglich temporär, bzw. er ist nicht immer abrufbar.

Frau Hellers Erzählung steht hier exemplarisch für eine Erfahrung, die mehrere Zeitzeug_innen in den Interviews beschreiben: Der öffentliche Diskurs um die Aufarbeitung der Heimgeschichte weckte das Interesse, sich mit der eigenen Vergangenheit zu beschäftigen und auf zuständige Institutionen zuzugehen. Weitere Gründe, die damit in Zusammenhang stehen können, sind die Hoffnung auf Entschädigungszahlungen sowie aktuell benötigte Unterlagen wie z.B. Zeugnisse. Das Ergebnis der Bemühungen um die über die eigene Person verfassten Schriftstücke ist oftmals enttäuschend. So konnten teilweise die Dokumente nicht aufgefunden werden oder es wurde der Zugang verwehrt bzw. erst, wie im Folgenden beschrieben, mit anwaltlicher Unterstützung gewährt. Welche Erfahrungen diejenigen gemacht haben, die sich Akteneinsicht verschaffen konnten, und welche Bedeutung den Dokumenten im Nachhinein zukommt, wird nachfolgend herausgearbeitet.

\section{Die Akteneinsicht}

Der Zeitzeuge Martin Neuhoff hat ebenfalls im Zuge des verstärkten medialen Diskurses um die Heimerziehung der 1950er und 1960er Jahre von der Möglichkeit erfahren, die damals angefertigten Dokumente einzusehen und er hat sein Recht auf Akteneinsicht kurz danach eingefordert. Obwohl die Akteneinsicht Betroffenen institutionell ermöglicht werden soll, stößt das Vorhaben oftmals auf bürokratische Widerstände. Dies geschieht beispielsweise wenn einige aufgrund der in den Akten enthaltenen Informationen und Belege sowie der teilweise hohen Brisanz, die die Dokumente für sie haben, diese nicht nur lesen, sondern auch besitzen möchten, um z.B. weiter recherchieren zu können. Davon erzählt auch Herr Neuhoff: ,und dann wollte man mir sie nit aushändigen dann hab ich $n$ Anwalt beordert dann haben sie 
also erst die Akte ausgehändigt“". Herr Neuhoff kann also erst mit der Hilfe eines befreundeten Anwalts durchsetzen, dass er Kopien von den über ihn angelegten Dokumenten machen kann. Wie wichtig es für ihn ist, die Dokumente vollständig zu besitzen, wird im folgenden Zitat deutlich: „da hab ich zur Sicherheit alles kopiert zweimal sogar und dann hab ich s Original wiedergegeben aber ich hab alles jetzt kopiert ich hab sie alle".

Warum es für Herrn Neuhoff so wichtig ist, die Akte bzw. Kopien davon dauerhaft verfügbar zu haben, lässt sich nachvollziehen, wenn er beschreibt, was der Inhalt für ihn bedeutet:

alles da alles A bis $\mathrm{Z}$ da da steht dann alles drin aber ich hab jetz die Antworten das war mir jetz wichtig und das is auch wichtig wo man sagen muss so weh s auch tut aber du hast auf viele Fragen ne Antwort und das is das was wiederum einen aufbaut zu lernen das is nich deine Schuld man - man gibt sich ja selbst die Schuld dafür hast du dich selbst fast geschämt weil du geboren worden bist

Herr Neuhoff erzählt hier, dass er in der Akte die Antworten auf seine offenen Fragen gefunden hat. Ihm scheinen somit mit der Akteneinsicht alle wichtigen Informationen zur Verfügung zu stehen, nach denen er gesucht hat. Das Lesen beschreibt er als schmerzhaft und gleichzeitig befreiend, da es ihn von einer Schuld befreit, die er sich zuvor selbst gegeben hat. Herr Neuhoff erfährt durch die Akteneinsicht u.a. von einer starken Vernachlässigung und Gefährdung durch seine Mutter kurz nach seiner Geburt. Diese und weitere Informationen helfen ihm, Teile seiner Biografie zu rekonstruieren, woraus er Erklärungen für sein eigenes Gewordensein ableitet. Der Akteninhalt entlastet ihn und stößt einen Lernprozess an, in welchem sich die selbst zugeschriebene Schuld an seinem Ich-Sein, die mit Scham verbunden ist, in eine Schuld transformiert, die nun anderen zugeordnet werden kann. Herr Neuhoff scheint den Inhalt der Akte als glaubhaft einzuordnen und beruft sich während des Interviews mehrmals auf diesen als Belegreferenz.

Auf die Frage, ob es eine gute Entscheidung gewesen sei, die Akte zu lesen, antwortet Herr Neuhoff:

das war ne Entscheidung weil - nee ich will mal so sagen man muss den Leuten auch mal klarmachen es hat wehgetan ich sag Ihnen ehrlich wie ich die Akte gelesen hab und bin mit der Straßenbahn wieder zu mir heim gefahren ich hab da gehockt mir sind da so die Tränen gekommen. Ich hab das verarbeiten müssen ich habs verarbeiten müssen

Herr Neuhoff leitet seine Antwort mit der Schilderung eines dringenden Bedürfnisses ein, Menschen darüber aufzuklären, wie schmerzhaft die Akteneinsicht für ihn war, und erzählt möglicherweise deshalb von seiner emotionalen Reaktion in der Straßenbahn, die er scheinbar nicht kontrollieren konnte und die ihn unvorbereitet traf. Der Akteninhalt muss laut Herrn Neuhoff erst verarbeitet werden, was darauf hindeutet, dass er durch die Lektüre Informationen bekommen hat, die entweder neu für ihn waren oder möglicherweise über die lange Zeitspanne zwischen dem Heimaufenthalt und der Akteneinsicht verdrängt wurden. Die Beschäftigung damit erscheint anstrengend und bedarf kräftezehrender psychischer Arbeit. 
Auch für die Zeitzeugin Jutta Kaiser bergen Dokumente aus der Zeit, die sie in Fürsorgeinstitutionen verbracht hat, Informationen, die Aufklärung über ihre Familienverhältnisse geben. Sie erzählt im Interview, dass sie sich im Alter von achtzehn Jahren selbst in ein Heim einweist, um der Gewalt durch die Mutter zu entkommen. In der Akte hat sie Briefe gefunden, die die Mutter damals an das Heim geschrieben hat:

jetzt habe ich die Briefe alle gelesen die hat sowas wie gelogen es is unglaublich ne ich weiß nich warum ich mich trotzdem immer wieder auch wundere ne man wundert sich trotzdem ne weil dann liegt ja auch so viel Jahrzehnte dazwischen man hat Distanz und dann auf einmal liest man in den Akten ihre Briefe was sie da gelogen hat

Aus der Passage geht hervor, dass Frau Kaiser sich zwar in ihrer Meinung über ihre Mutter durch deren Briefe bestätigt findet, dass sie aber gleichzeitig auch über den Inhalt sowie ihre eigene Reaktion überrascht ist. Die beschriebene Verwunderung führt sie darauf zurück, dass sie durch die vergangene Zeit Distanz aufgebaut hat. Hier ist denkbar, dass sie die Distanz zur Mutter oder allgemein zur Zeit der Entstehung der Dokumente meint. Diese über die Jahre aufgebaute Distanz scheint mit der Aktenlektüre schlagartig zu verschwinden und sie findet sich plötzlich in der Vergangenheit wieder, in der ihre Mutter Briefe mit Lügen über sie an die Heimleitung schickt.

Durch ihre Säuglingsakte findet Frau Kaiser außerdem heraus, dass ihre Mutter damals eine Geschlechtskrankheit hatte:

das is auch dann nicht leicht zu lesen [...] ja ich hab n Schlag gekriegt die hätte mich anstecken können die hat mich aber nicht angesteckt das sind so Dinge die müssen se erstmal verdauen

Der Akteninhalt hält demnach Überraschungen für Frau Kaiser bereit, mit denen sie nicht gerechnet hat und die einen Prozess der aktiven Verarbeitung erfordern. Das Gelesene deckt sich aber im Kern mit Jutta Kaisers Wahrnehmung und erscheint somit glaubwürdig. Der Akteninhalt und die eigene Erinnerung können allerdings auch in Widerspruch treten, wie es sich in folgender Sequenz andeutet, in der Frau Kaiser auf die Frage antwortet, wie es für sie war, ihre Akte einzusehen:

man guckt sich erstmal spontan an dann legt man sie wieder weg ne und grübelt grübelt grübelt ne das sind so zum Beispiel so Kleinigkeiten die mich aber äh ärgern weil ich weiß dass s nich richtig is

Jutta Kaiser erzählt hier, dass sie nach dem ersten spontanen Leseeindruck ins Grübeln kommt, da ihr Aussagen auffallen, die sie aus der Retrospektive ärgern, weil sie mit großer Gewissheit nicht der Wahrheit entsprechen, die sie erinnert. Frau Kaiser fallen dazu im Verlauf des Interviews zahlreiche Beispiele ein, die sich meist auf Akteneinträge beziehen, die über ihr Verhalten gegenüber den anderen untergebrachten jungen Frauen berichten. Im folgenden Zitat geht es um einen Eintrag, laut dem Frau Kaiser im Heim Untergebrachte mit Zigaretten versorgt hätte und sich in einem nachfolgenden Gespräch mit einer Erzieherin geweigert hätte, andere zu verraten: 
dann steht in der Akte drin äh ich hätte Mädels mit Zigaretten versorgt und ich hätte da zum Gespräch gemusst und hätte der Erzieherin gesagt ich verpfeif die Mädchen nich allein der Ausdruck stimmt schon nicht ich hätte niemals gesagt verpfeife äh ich hätte petzen gesagt

Frau Kaiser leitet die zitierte Passage mit einer Erzählung über ein solidarisches Miteinander in der Gruppe der Heimzöglinge ein, das durch kollektives Rauchen gestärkt wird. Es ist zwar möglich, dass die in der Akte beschriebene Versorgung der anderen jungen Frauen mit Zigaretten stattgefunden hat, Frau Kaiser beschreibt dies als einmalige Aktion, die Beschreibung des anschließenden Gesprächs mit der Erzieherin wird von Frau Kaiser jedoch auf Grundlage der Wortwahl der schreibenden Person als ,glatte Lüge“ enttarnt:

aber ich weiß wer diese Gaunersprache hatte weil die eine die Schwester Ursula die mich auch mit ihrer Sprache immer irritiert hat [...] war ich auch immer $n$ bisschen angeekelt von der Frau weil ich das nicht so mochte ne und dann steht hier da drin ich hätts gesagt und das stimmt niemals das weiß ich niemals niemals und ich müsste mich auch an das Gespräch erinnern ganz im Gegenteil ich war immer heilfroh dass es nicht aufgeflogen ist ne also das ist ne glatte Lüge

An dieser Sequenz fällt zunächst die hohe Emotionalität auf, mit der Frau Kaiser erzählt. In der Wiederholung der Verneinung ,niemals“ wird deutlich, dass sie über den damaligen Eintrag wütend und entsetzt ist, weil er ganz und gar nicht ihrer Erinnerung entspricht. Aus der Retrospektive ist sie sich sicher, dass das Gespräch zwischen der Erzieherin und ihr gar nicht oder zumindest nicht so wie in der Akte beschrieben stattgefunden hat. Die im Interview spürbar werdenden Emotionen resultieren nicht nur aus der Feststellung, dass in den Dokumenten gelogen wurde, sondern sind auch stark an Frau Kaisers Erinnerung an die Verfasserin geknüpft. Anhand der verwendeten „Gaunersprache“ identifiziert sie die Schreibende als Schwester Ursula, mit der sie ein Gefühl des Ekels assoziiert, das sie an deren Ausdrucksweise festmacht. Ein weiterer Eintrag von dieser Person verärgert Frau Kaiser zusätzlich:

ne weitere Lüge ich hätte mich von den Mädchen bedienen lassen das hat mich wirklich äh äh die Frau kann froh sein dass sie nicht mehr lebt weil das ist ne Frechheit

Frau Kaiser lässt zwar offen, was passieren würde, wenn die Verfasserin des Eintrages noch leben würde, die zitierte Sequenz suggeriert allerdings, dass Frau Kaiser das über sie Geschriebene nicht so stehen lassen und die Verfasserin möglicherweise aufsuchen und zur Rede stellen würde. Die Behauptung des Akteneintrags empört Frau Kaiser besonders, weil sie sich und ihr damaliges Handeln ganz anders in Erinnerung hat. Im Anschluss an die zitierte Passage erzählt Frau Kaiser ausführlich, warum das über sie Festgehaltene nicht der Wahrheit entsprechen kann. Zum einen würde sie eine, die sich bedienen lässt, als Verräterin ansehen und zum anderen habe sie es nicht nötig gehabt, da sie Geld genug gehabt hätte. Vielmehr hätte sie die anderen jungen Frauen unterstützt. Im Verlauf des Interviews beschreibt sie sich selbst 
häufig als kümmernde, helfende und vor allem solidarische Ansprechpartnerin für die im Heim untergebrachten Frauen. Die in der Akte beschriebene Person scheint so gar nicht Frau Kaisers Selbstbild zu entsprechen und die Erinnerung an die Aktenlektüre veranlasst Frau Kaiser im Interview in eine Verteidigungshaltung zu gehen und der Interviewerin vor dem Hintergrund ihrer Arbeitsbiografie, ihres großen Engagements für andere ehemalige Heimkinder und persönlicher Eigenschaften beweisen zu wollen, dass sie so nicht gewesen sein kann. Die folgende Erzählsequenz, in der sie von ihrem Einsatz für Betroffene erzählt, die sie bei ihrer späteren Erwerbsarbeit in einer Psychiatrie kennengelernt hat, illustriert dies ebenfalls anschaulich:

da hatte ich auch drei ehemalige Heimkinder die ich dann auch bei der Entschädigung geholfen hab ne und äh vier waren es glaub ich sogar ne und das macht man nicht wenn man so steht wie die da einen beschreiben da bin sauer ne ich bin nicht eigentlich da spür ich mich eigentlich noch als Achtzehnjährige und würde der gerne mal eine auf die Kinnlade hauen

Bei diesem Zitat fallen zunächst die unterschiedlichen Zeiträume auf, die Frau Kaiser beim Erinnern und Erzählen durchschreitet: Die geleistete Unterstützung für Betroffene ist nah an der Gegenwart, während die Akteneinträge vor mindestens vierzig Jahren, also weit in der Vergangenheit zurückliegend, erstellt wurden. Bei der Lektüre aus der Retrospektive und dem Erzählen darüber scheinen die Zeiten zusammenzufallen, wenn die Interviewte sagt, dass sie so wütend über das Geschriebene ist, dass sie sich ,noch als Achtzehnjährige“ empfindet und dies mit einer adoleszenten Gewaltfantasie unterstreicht. Ein Teil der jungen Frau, die sie einst gewesen ist, scheint durch die Aktenlektüre wieder präsent und für sie selbst spürbar zu werden, was sich im gesamten Interview auch durch ihre teilweise jugendsprachliche Ausdrucksweise zeigt. Die Argumentation, dass sie als Jugendliche nicht so gewesen sein kann, wie die Dokumente es beschreiben, weil ihr Handeln in der nahen Vergangenheit dem zuwiderlaufen würde, kann verschieden gedeutet werden: Der verletzende Akteninhalt macht diese Argumentation notwendig und es ist somit vorstellbar, dass das Geschriebene einen hohen Rechtfertigungsdruck vor der Interviewerin oder auch vor sich selbst erzeugt. Denn zugleich könnte der Inhalt Frau Kaiser verunsichern und eine Identitätskrise auslösen, da sie sich möglicherweise auch fragt, ob Teile des Geschriebenen nicht doch wahr sein könnten. Dies würde ihre Identität, ihre bisherige Selbstkonstruktion infrage stellen und sie veranlassen, ihre Biografie anders zu deuten. Diese Vermutung könnte Frau Kaisers hochemotionale Abwehrreaktion dem Akteninhalt gegenüber erklären.

Hinzu kommt die Unveränderlichkeit der Dokumente:

Das steht da schwarz auf weiß ich kann die Worte nicht ausradieren ich kann nicht die Situation erklären hab schon überlegt ob ich die nehme und dann mal n Statement dazu mache ,bist du denn bekloppt die Alte is schon längst tot die Zeit ist vorbei“" ne aber es äh hätt nich gedacht dass mich das innerlich noch so bewegt ne dabei kann es sogar sein dass die äh die sehen das ganz anders wie ich ne ,ich kenn doch die Jutta ich weiß doch dass die da Scheiße geschrieben haben" oder so das nützt da gar nix ne ne das nochmal da wirklich äh nochmal so ne zusätzliche Demütigung ne da hat man sich vierzig Jahre was erkämpft 
und mit so einer Akte können sie in zwei Minuten genauso einen extremen Rückfall kriegen dann sind sie wieder achtzehn und nix anderes und das ist etwas was man nicht äh über die Zeit wegnehmen kann ne das ist etwas was einfach ähm da sind sie achtzehn das steht da das kann ich nicht korrigieren

Aus dieser Sequenz spricht eine große Ohnmacht gegenüber der Wirkmacht der über vierzig Jahre alten Dokumente. Die damaligen Worte lassen sich nicht mehr entfernen und auch nicht korrigieren. Für ein öffentliches Dementi ist die Zeit für Frau Kaiser schon zu weit fortgeschritten, da die Diakonisse, die die Texte verfasst hat, bereits verstorben ist. Die Zitation der eigenen Überlegungen wirkt wie eine strenge Ermahnung an sich selbst, den Gedanken an ein Statement fallen zu lassen, da sie sonst an ihrer geistigen Gesundheit zweifeln müsste. Damit bleibt Frau Kaiser jede Möglichkeit, sich mit der Autorin des Akteneintrags auseinanderzusetzen, versagt und auch wenn ihr das rational kognitiv bewusst ist, verspürt sie den Impuls auf das Geschriebene zu reagieren. Eine derart emotionale Reaktion auf die Aktenlektüre hatte Frau Kaiser von sich selbst nicht erwartet und sie erklärt sich diese durch die zusätzliche Demütigung, die der Akteninhalt für sie bedeutet. Auch wenn Personen, die sie kennen, das Geschriebene schnell als unwahr entlarven könnten, ändert das für ihr Empfinden nichts. Die Aktenlektüre scheint Frau Kaiser emotional direkt in die Vergangenheit zu versetzen und die damaligen Kränkungen wieder wachzurufen und fortzuschreiben. Frau Kaiser beschreibt diesen psychischen Prozess als „extremen Rückfall“. In der Medizin steht ein Rückfall für das Wiederauftreten einer scheinbar überstandenen Krankheit. Bei Frau Kaiser könnte ein Rückfall die Wiederkehr von bereits behandelten, in der Kindheit und Jugend erlebten Traumata bedeuten. Die Akte hat somit das Potenzial, Betroffene innerhalb kürzester Zeit mit lange vergangenen, schmerzhaften Erfahrungen zu konfrontieren und diese auch gegenwärtig wieder fühlbar zu machen. Die mehr als vierzig Jahre zwischen damaliger Erfahrung und retrospektiver Erinnerung werden bedeutungslos oder ,schmelzen auf einmal zusammen“, wie es Frau Kaiser an anderer Stelle des Interviews beschreibt, als würden sie sich in Luft auflösen und wären damit nicht mehr existent. Zurück bleibt eine Jugendliche, die sich durch die über sie verfassten Dokumente erneut der Willkür des Heimpersonals ausgeliefert sieht. Die Erzählerin fühlt sich in die Vergangenheit zurückversetzt und wird wieder zu der jungen Frau von damals. In dieser Position hat sie keine Möglichkeit, Einfluss auf die Dokumentation des Erziehungspersonals zu nehmen. Auch wenn eine Korrektur des Akteninhalts ausgeschlossen ist, entwickeln Frau Kaiser und andere Betroffene Fantasien und Strategien für den zukünftigen Umgang mit dem Akteninhalt, wie im Folgenden gezeigt wird.

\section{Handlungs(un)möglichkeiten im Umgang mit der Akte}

Für Jutta Kaiser ist die Beschäftigung mit dem Akteninhalt noch nicht abgeschlossen und sie möchte sich dem wieder widmen, wenn sie „mal mehr Ruhe“ hat. Sie hat für sich aber bereits mehrere Ideen und Handlungsmöglichkeiten entwickelt, die im Folgenden dargestellt und analysiert werden. Anhand des untenstehenden Zitates wird zunächst noch einmal deutlich, wie schmerzhaft die Lektüre für sie war: 
also sowas aus der Luft gegriffen und das hat mir wehgetan weil ich dachte da hätt ich so s Gefühl da hätt ich gern nochmal ne Gerichtsverhandlung über so viel Lügen ja weil weil man in eine in was für eine Rolle wird man da gedrängt und das bin ich nicht ganz im Gegenteil ne

Die festgeschriebene Unwahrheit wiegt für Frau Kaiser so schwer, dass sie sich eine durch eine Gerichtsverhandlung herbeigeführte Gerechtigkeit wünscht, die das durch die Dokumente konstruierte Bild richtigstellt. Die Akte drängt sie in eine Rolle, die ihr nicht entspricht und konträr zu ihrer Selbstkonstruktion steht.

da fühl ich mich echt entwertet durch diese Akte da hab ich schon überlegt ich möchte das die vernichtet wird weil die wird eh kein Mensch verstehen ne und das das mmh irgendwie hätt ich da so das Bedürfnis das richtig zu stellen dass ich nicht so bin

Der Angriff auf die eigene Person durch die Akte ist für Frau Kaiser so gravierend, dass sie sich nicht nur abgewertet oder falsch dargestellt wahrnimmt, sondern eine „Entwertung“ erfährt. Dieser Begriff zielt auf eine Herabwürdigung oder sogar eine Negierung ihrer Person. Aus der Annahme, dass niemand den Inhalt der Akte richtig deuten kann, resultiert für sie der Wunsch, dass die Dokumente vernichtet werden. Gleichzeitig ist es ihr ein großes Anliegen, den Eindruck, den die Akte bei anderen hinterlassen könnte, zu korrigieren. Da ihr klar ist, dass sie das nicht immer tun kann, ist die einzige Möglichkeit, das Geschriebene wenigstens auf dem Papier auszulöschen. Diese Überlegungen konkretisiert sie im weiteren Verlauf des Interviews:

[...] dann denk ich da $\mathrm{n}$ paar Leute kennen mich und die lesen das was sollen die über mich denken dann war ich ne Verräterin war ich aber nich ne is völlig unlogisch wer mich kennt [...] der weiß dass ich n Mensch bin der wirklich echt äh für alles Unrecht eintritt wenn einer das versteht bin ich das und dafür bin ich dort auch bekannt [...] und dann kommt so ne gehässige Diakonisse die mich damals schon gequält hat und schreibt da so $n$ Scheiß [...] ich würde gerne mal jemandem meine Akte zeigen aber äh kann ich nicht weil ich müsste ständig mich rechtfertigen [...] welche Hässlichkeit die eine Erzieherin über mich geschrieben hat darüber werd ich nie mit jemand reden können und das ei ärgert mich eigentlich dass ich nicht so viel Distanz hab ,sag seht mal wat die da fürn Scheiß geschrieben hat" andere wissen ja dass das Scheiß is und schämen sich trotzdem leider ne also werd ich mal meine Akte wenn irgendwann bevor ich sterbe vernichten oder so ne das sie einfach nicht an die Öffentlichkeit ne [...] werd ich mich eines Tages entscheiden müssen das $\mathrm{zu}$ vernichten damit das nicht noch äh nach meinem Tod jemand anders in die Hände bekommt ne auch Schade drum

In dieser längeren Interviewsequenz werden Frau Kaisers Befürchtungen bezüglich des Akteninhalts und des zukünftigen Verbleibs der über sie angefertigten Dokumente noch einmal greifbar. Ihre Sorge bezieht sich besonders darauf, dass andere Menschen sie als „Verräterin“ lesen könnten, da die Verfasserin des Eintrags sie aus ihrer heutigen Wahrnehmung in der Akte als solche konstruiert hat. Sie selbst sieht 
sich jedoch als eine Person, die für Gerechtigkeit sorgt und geht davon aus, dass sie für diese Eigenschaft auch bei anderen bekannt ist. Daraus resultiert, dass der Akteninhalt als ,unlogisch“, also folgewidrig, vor dem Hintergrund des Wissens über sich selbst wahrgenommen wird. In dieser Passage spricht Frau Kaiser über die damals schreibende „Diakonisse“ als wäre sie eine präsente Person und hätte den Akteneintrag erst kürzlich angefertigt. Sie hat sie damals schon gequält und tut dies mit den vor über vierzig Jahren verfassten Schriftstücken bis in die Gegenwart. Wie bereits oben erläutert, scheint die inzwischen vergangene Zeit mit all ihren biografischen Erfahrungen zu verschwinden und Frau Kaiser fühlt sich wieder als Achtzehnjährige, die nichts gegen die aus der Retrospektive als willkürlich wahrgenommenen Einträge unternehmen kann. Dieses Ineinanderfallen verschiedener Temporalitäten untermauert die zuvor angedeutete Idee, dass der Akteninhalt einen hohen Rechtfertigungsdruck erzeugt. Der Wunsch, anderen Menschen die Akte zu zeigen, resultiert möglicherweise aus einem Bedürfnis der Richtigstellung, aber auch der Selbstvergewisserung heraus und ist von ambivalenten Gefühlen begleitet. Auch wenn die Schriftstücke Frau Kaiser helfen könnten, anderen Personen Ausschnitte ihrer Vergangenheit näher zu bringen, und ein Austausch darüber Frau Kaisers (Selbst-)Einschätzungen stärken könnten, ist die Angst vor einem möglichen Missverstehen und einer damit einhergehenden Stigmatisierung größer. Sie befürchtet unter Rechtfertigungsdruck zu geraten, dem sie nicht standhalten kann, weil das Geschriebene eine solche „Hässlichkeit“ ist, dass es zu schmerzhaft wäre, darüber in einen verbalen Austausch zu gehen. Frau Kaiser reflektiert, dass sie die Distanz, die sie sich für sich selbst wünscht und die nötig wäre, um ihre Position gegenüber dem Dokumenteninhalt deutlich zu machen und sich anschließend dem Urteil anderer zu stellen, nicht aufbauen kann. Trotzdem weiß sie, dass das Geschriebene ,Scheiß“ ist. In dieser affektiven Ausdrucksweise zeigt sich eine hohe Emotionalität, die auch ein Ohnmachtsgefühl bezogen auf die über ihren Tod andauernde Macht der Schriftstücke beinhalten könnte. Auch andere würden wissen, dass Akteninhalte dreiste Lügen darstellen, sie würden sich aber trotzdem für das über sie Verfasste schämen. Mit den anderen sind hier wahrscheinlich weitere Betroffene der Heimerziehung gemeint, von denen sie sich aber zu unterscheiden scheint. Hier verschwimmt Eigenes mit Fremden und die von anderen empfundene Scham könnte auch ein Teil von ihr sein. Dieser Teil wäre aber in Zusammenhang mit ihrer vorangegangenen Argumentation und ihrer Selbstkonstruktion kaum vereinbar und wäre somit nur schwerlich zu versprachlichen. Dennoch ist es unter anderem die Scham, die ihr die Entscheidung, die Akte vor dem eigenen Tod zu vernichten, näherbringt. Sie möchte damit verhindern, dass die Dokumente an die Öffentlichkeit oder überhaupt in andere Hände geraten könnten. Hierbei scheint der Gedanke, das unveränderlich Festgeschriebene für die Welt nach ihr unkommentiert stehen lassen zu müssen, unerträglich und eine Lösung nur in der Vernichtung zu liegen. Besonders interessant ist, dass sie die Zerstörung der Dokumente trotzdem bedauern würde. Warum Frau Kaiser eine Vernichtung ,schade“ fände, führt sie zwar hier nicht weiter aus, dieser Ausdruck des Bedauerns verweist aber auf den hohen Wert, den auch andere Zeitzeug_innen den Akten beimessen. In Frau Kaisers Ringen um Vernichtung oder Weitergabe der Schriftstücke zeigt sich, dass der Umgang mit den Dokumenten von einer nur schwer auszuhaltenden Ambivalenz begleitet ist, die sich nicht einfach auf- 
lösen lässt, sondern in Prozessen der Aufarbeitung und Verarbeitung allgegenwärtig zu sein scheint.

Vor dem Hintergrund der Erzählung von Jutta Kaiser und den Erzählungen anderer Betroffener der Heimerziehung der 1950er und 1960er Jahre wird die bis heute andauernde Bedeutung der Akte nachvollziehbar. Zugleich kristallisiert sich heraus, dass diese Bedeutung verschieden ausgestaltet ist. So erzählen mehrere Zeitzeug_innen, dass sie die Akte zur Vorbereitung auf das biografische Interview genutzt haben. Auch Vorträge über ihre Heimerfahrung, die Betroffene vor Schulklassen oder anderen Gruppen halten, werden häufig unter Zuhilfenahme der Dokumente ausgearbeitet. Im Interview und im Gespräch mit anderen dient die Akte nicht selten als Beleg für erfahrenes Unrecht und konnte bereits Betroffenen helfen, ihr Recht auf Entschädigung geltend zu machen. Besonders wichtig ist für die meisten ehemals im Heim Untergebrachten die Rekonstruktion der eigenen Biografie. Vor allem denjenigen, die bereits als (Klein-)Kinder in Heime eingewiesen wurden und keine oder nur wenig Bezugspersonen außerhalb der Institution hatten, sind heute auf die damals erstellten Unterlagen angewiesen, wenn sie etwas über ihre Kindheit und Jugend erfahren möchten.

\section{Fazit und Ausblick}

Wie gezeigt werden konnte, bedeuten der Weg zur Akteneinsicht sowie die anschließende Beschäftigung mit dem Inhalt eine große emotionale Herausforderung für Zeitzeug_innen der damaligen Fürsorgepraxis. Die Lektüre, der über sie angefertigten Schriftstücke, kann die erlebte Vergangenheit mit den dazugehörigen Akteur_innen nicht nur wieder fühlbar und gedanklich präsent machen, sondern auch in einem ganz neuen Licht erscheinen lassen kann. So verwundert es nicht, dass die Akteneinsicht höchst ambivalente Gefühle und Gedanken auslöst. Zum einen werden sich aus dem Akteninhalt wichtige Informationen zur Rekonstruktion und zum Verstehen der eigenen Biografie sowie Belege für erfahrenes Unrecht und damit ein Recht auf Wiedergutmachung versprochen. Zum anderen enthält die eigene Akte kränkende Aufzeichnungen, die mit der eigenen Erinnerung in heftigen Widerspruch treten. Die Angst vor erneuter Stigmatisierung sowie die Gefahr von Retraumatisierung ${ }^{5}$ scheint dem Prozess der Aufarbeitung inhärent.

Die Akte hat für die Zeitzeug_innen zwar das Potenzial, Wissenslücken in der eigenen Erinnerung aufzufüllen, das in den Akten im Zuge bürokratischer Prozesse hergestellte institutionsspezifische Sonderwissen, kann dem Wunsch nach Aufklärung jedoch nur bedingt nachkommen. Zäsur und Kontrolle von Wissen sind charakteristisch für Verwaltungsdokumente (vgl. Muckel 2000). Dadurch sind Rekonstruktionen von Lebensgeschichten nur bruchstückhaft und vor dem Hintergrund der subjektiven Wahrnehmung sowie dem verwaltungsrechtlichen Auftrag der Schreibenden möglich. Diese so konstituierten biografischen Teilstücke finden Eingang in die Narration der Interviewten und verwickeln sich dort mit der eigenen Erinnerung

\footnotetext{
${ }^{5}$ Zur Gefahr der Retraumatisierung durch die Aktenlektüre, aber auch durch biographische Interviews siehe Apel und Eckhardt (2019).
} 
sowie mit den sprachlichen und visuellen Bildern des öffentlichen Diskurses. Nach Rosenthal (2010, S. 198) ,bestimmt die Gegenwart des Erzählens [...] den Rückblick auf die Vergangenheit und erzeugt eine jeweils spezifische erinnerte Vergangenheit.“ Die Erinnerung an die Vergangenheit wird demnach von gegenwärtigen Erfahrungen geprägt und verändert. Wenn sich Betroffene also in irgendeiner Form ${ }^{6}$ für eine Auseinandersetzung mit der eigenen Vergangenheit entschieden haben, den gesellschaftlichen Diskurs verfolgt und/oder ihre Akte eingesehen haben, kann davon ausgegangen werden, dass ihre Erzählung vorher eine andere gewesen wäre. Die Aktenlektüre lässt Vergangenheit und Gegenwart zusammenfallen und verändert damit nicht nur vorherige Erinnerungen, sondern auch die Antizipation von zukünftigen Handlungsoptionen.

Der Verantwortungsdruck gegenüber nachfolgenden Generationen, die damaligen Geschehnisse aufzuarbeiten, wurde mit der Zunahme der öffentlichen Aufmerksamkeit für das Thema verstärkt, wie am Beispiel von Gisela Heller gezeigt werden konnte. Deswegen und aufgrund der durch die Initiative des Runden Tisches Heimerziehung geschaffenen Möglichkeiten könnte bei vielen der Wunsch entstanden oder gewachsen sein, die eigene Akte einzusehen. Für diejenigen, die den erforderlichen Mut aufgebracht haben, diesem Bedürfnis zu folgen und die erfolgreich bei der Beschaffung der Dokumente waren, bedeutet der Akteninhalt Verschiedenes: Antworten auf lange gestellte Fragen, die falsche oder verdrehte Darstellung von Geschehnissen sowie Eigenschaften der eigenen Person oder die Möglichkeit, erfahrenes Unrecht zu beweisen. Die Materialität der Akte kann als bedrohlich empfunden werden, weil die Weitergabe der dort enthaltenen Informationen nur begrenzt kontrolliert werden kann. Die Akte wird jedoch als hilfreich eingestuft, wenn sie als Belegrundlage und zur Veranschaulichung von Erzählungen dient. Da die Schriftstücke nicht selbsterklärend sind, können sie allerdings einen hohen Rechtfertigungsdruck für die Betroffenen erzeugen.

Mit der Gewährung von Akteneinsicht müssen die Betroffenen nicht nur selbstbestimmt entscheiden, ob sie die Dokumente sehen wollen, sondern auch wie sie diese in die fragilen Konstrukte der eigenen Biografie einordnen. Da die spezifische Perspektive der Dokumente eine objektivierende ist und die Subjekte nicht als solche fassen kann, sondern sie vorrangig vor dem Hintergrund der Interessen der Institution(en) konstruiert (Muckel 1997), lässt sich grundsätzlich fragen, inwieweit Verwaltungsdokumente biografische Aufarbeitungsprozesse sinnvoll begleiten können. Die Zeitzeug_innen erkennen zwar teilweise Logiken der damaligen Aktenführung, geraten aber dennoch bei der Reflexion auf das eigene Gewordensein in existenzielle Konflikte, wenn sie zurückliegende Erinnerungen und gegenwärtige Erfahrungen zur Deckung bringen wollen und dabei auf bürokratische Dokumente vertrauen wollen oder müssen. Die Narrationen, die dabei entstehen, sind dadurch gefärbt und weisen Spuren verschiedener politischer und gesellschaftlicher Diskurse sowie die einer institutionalisierten Sprache auf, die es bei der Auswertung dieser Daten zu berücksichtigen und mit der Erfahrung der Akteneinsicht in Beziehung zu setzen gilt.

\footnotetext{
6 z.B. biographisches Schreiben oder Erzählen, Psychotherapie, Teilnahme an Diskussionen in Internetforen oder Veranstaltungen.
} 
Funding Open Access funding provided by Projekt DEAL.

Open Access Dieser Artikel wird unter der Creative Commons Namensnennung 4.0 International Lizenz veröffentlicht, welche die Nutzung, Vervielfältigung, Bearbeitung, Verbreitung und Wiedergabe in jeglichem Medium und Format erlaubt, sofern Sie den/die ursprünglichen Autor(en) und die Quelle ordnungsgemäß nennen, einen Link zur Creative Commons Lizenz beifügen und angeben, ob Änderungen vorgenommen wurden.

Die in diesem Artikel enthaltenen Bilder und sonstiges Drittmaterial unterliegen ebenfalls der genannten Creative Commons Lizenz, sofern sich aus der Abbildungslegende nichts anderes ergibt. Sofern das betreffende Material nicht unter der genannten Creative Commons Lizenz steht und die betreffende Handlung nicht nach gesetzlichen Vorschriften erlaubt ist, ist für die oben aufgeführten Weiterverwendungen des Materials die Einwilligung des jeweiligen Rechteinhabers einzuholen.

Weitere Details zur Lizenz entnehmen Sie bitte der Lizenzinformation auf http://creativecommons.org/ licenses/by/4.0/deed.de.

\section{Literatur}

Apel, Magdalena, und Lina Eckhardt. 2019. Die Akte in der Fürsorgeerziehung der 1950er und 1960er Jahre - Institutionelle Praxis und biografische Verarbeitungsprozesse. In Geschlossene Institutionen - Theoretische und empirische Einsichten, Hrsg. Anke Neuber, Franz Zahradnik, 91-106. Weinheim: Beltz Juventa.

Bereswill, Mechthild, und Patrik Müller. 2018. Die administrierte Biografie in der Heimerziehung der 1950er bis 1970er Jahre. In Verwaltete Biografien, Hrsg. Elisabeth Schilling, 3-26. Wiesbaden: Springer.

Bereswill, Mechthild, Theresia Höynck, und Karen Wagels. 2013. Heimerziehung 1953-1973 in Einrichtungen des Landeswohlfahrtsverbandes Hessen. Bericht zum Interdisziplinären Forschungs- und Ausstellungsprojekt. www.lwv-hessen.de/geschichte-gegenwart/heimerziehung/forschung-ausstellung. html. Zugegriffen: 9. Apr. 2019.

Muckel, Petra. 1997. Der Alltag mit Akten - psychologische Rekonstruktionen bürokratischer Phänomene. Eine empirische Untersuchung in verschiedenen Institutionen. Aachen: Shaker.

Muckel, Petra. 2000. Methodische Reflexionen zur Forschung in Institutionen - Phänomenspiegelungen als Erkenntnisinstrument. Forum Qualitative Sozialforschung/Forum: Qualitative Social Research, 1(2), Art. 22. www.qualitative-research.net/index.php/fqs/article/view/1091/2390. Zugegriffen: 9. Apr. 2019.

Oevermann, Ulrich, Tilman Allert, Elisabeth Konau, und Jürgen Krambeck. 1979. Die Methodologie einer ,objektiven Hermeneutik“ und ihre allgemeine forschungslogische Bedeutung in den Sozialwissenschaften. In Interpretative Verfahren in den Sozial- und Textwissenschaften, Hrsg. Hans-Georg Soeffner, 352-434. Stuttgart: Metzler.

Rosenthal, Gabriele. 2010. Die erlebte und erzählte Lebensgeschichte. Zur Wechselwirkung zwischen Erleben, Erinnern und Erzählen. In Subjekt-Identität-Person? Reflexionen zur Biographieforschung, Hrsg. Birgit Griese, 197-218. Wiesbaden: Springer VS.

Runder Tisch Heimerziehung. 2010. Abschlussbericht Runder Tisch „Heimerziehung der 50er und 60er Jahre“. www.fonds-heimerziehung.de/fileadmin/de.fonds-heimerziehung/content.de/dokumente/ RTH_Abschlussbericht.pdf. Zugegriffen: 9. Apr. 2019.

Zaft, Matthias. 2011. Der erzählte Zögling. Narrative in den Akten der deutschen Fürsorgeerziehung. Bielefeld: transcript. 